\title{
Dynamical Entropy of Quasi-local Algebras in Quantum Statistical Mechanics *
}

\author{
Yong Moon Park and Hyun Hye Shin \\ Department of Mathematics, Yonsei University, Seoul 120-749, Korea \\ Received April 19, 1991
}

\begin{abstract}
We study the dynamical entropy in the sense of Connes, Narnhofer, and Thirring of automorphisms on quasi-local algebras in quantum statistical mechanics. We extend their Kolmogorov-Sinai type theorem for AF-algebras to quasi-local algebras which are not necessarily AF-algebras.
\end{abstract}

\section{Introduction}

In their recent paper [5] Connes, Narnhofer and Thirring generalized the notion of a dynamical entropy introduced by Kolmogorov and Sinai $[8,15]$ for classical dynamical systems to the case of automorphisms of $C^{*}$-algebras invariant with respect to a given state, and they obtained a Kolmogorov-Sinai type theorem [5] for approximately finite (AF) algebras. The main purpose in this paper is to extend their result to quasi-local algebras in quantum statistical mechanics which are not necessarily approximately finite.

The concept of the Kolmogorov-Sinai (KS) entropy of measure preserving transformations became a key notion in ergodic theory [2] and also it allowed a formulation of the variational principle in statistical mechanics [14]. A quantum or non-commutative analogue of KS entropy was required for both to provide an important mathematical concept for quantum dynamical systems and to be applicable in quantum statistical mechanics. There have been several attempts to generalize the classical theory to non-commutative cases $[3,4,6,10]$. In order that a generalization is meaningful, it is natural to require that if it is restricted to classical systems, it must reduced to the KS entropy and that it has the continuity of the KS type $[8,15]$ to be able to compute the entropy. Due to much progress in quantum statistical mechanics [1,9], Connes, and Störmer were able to handle the

* Work supported in part by Korean Science Foundation and the Basic Science Research Program, Ministry of Education, 1991 
tracial state [3]. In [5] Connes, Narnhofer, and Thirring extended the tracial case to the non-tracial situation, and they also established the continuity of the KS type for AF-algebras. In order to apply Connes, Narnhofer, and Thirring (CNT) theory to unbounded spin systems and Bose particle systems in quantum statistical mechanics [1] one has to extend the continuity of the KS type to quasi-local algebras which are not $\mathrm{AF}$-algebras.

We organize the paper as follows: In Sect. 2 we review the definitions and the results on the CNT entropy [5] which will be used in the sequel. In Sect. 3, we list basic assumptions on quasi-local algebras (the tensor product property) and on the states (the locally normal property), and then give our main result. Section 4 is devoted to proof of the main result. As a simple application of our results we compute the dynamical entropy of space translations for product states of the unbounded quantum lattice system in Sect. 5 .

\section{Dynamical Entropy of $C^{*}$-Algebras}

As a preparation, we review the definition and some results on the dynamical entropy in [5] which are needed in the sequel. Throughout this section we consider a $C^{*}$-dynamical system $(\mathscr{A}, \theta, \phi)$, where $\mathscr{A}$ is a unital $C^{*}$-algebra, $\theta$ is an automorphism on $\mathscr{A}$ and $\phi$ is a state over $\mathscr{A}$ which is invariant with respect to $\theta$.

Let $\mathscr{A}$ be a finite dimensional $C^{*}$-algebra and let $\phi$ and $\psi$ be states on $\mathscr{A}$. The density operators corresponding to $\phi$ and $\psi$ are denoted by $\varrho_{\phi}$ and $\varrho_{\psi}$, respectively. The relative entropy for the states $\phi$ and $\psi$ is defined by

$$
S(\phi \mid \psi) \equiv \operatorname{Tr}\left(\varrho_{\psi}\left(\log \varrho_{\psi}-\log \varrho_{\phi}\right)\right),
$$

where $\operatorname{Tr}$ denotes the trace.

A completely positive unital map $\gamma$ between two unital $C^{*}$-algebras $\mathscr{A}$ and $\mathscr{B}$ is defined by a positive unital map such that the map $\gamma$ between $M_{n}(\mathscr{A})$ and $M_{n}(\mathscr{B})$ the $n \times n$ matrices with elements from $\mathscr{A}$ (respectively $\mathscr{B}),(\gamma(a))_{i j}=\gamma\left(a_{i j}\right)$, is positive for all $n$. With respect to composition they form a semigroup which contains *-homomorphisms. If $\mathscr{B} C \mathscr{A}$, a positive unital map $\gamma: \mathscr{A} \rightarrow \mathscr{B}$ with $\gamma\left(b_{1} a b_{2}\right)$ $=b_{1} \gamma(a) b_{2}, b_{i} \in \mathscr{B}, a \in \mathscr{A}$, is called a unital conditional expectation. The natural inclusions and unital conditional expectations are completely positive unital maps.

We recall the definition of the CNT entropy in [5]. Let $\mathscr{A}$ be a unital $C^{*}$ algebra, $\mathscr{N}_{1}, \ldots, \mathscr{N}_{k}$ finite dimensional $C^{*}$-algebras and $\gamma_{j}: \mathscr{N}_{i} \rightarrow \mathscr{A}$ a completely positive unital map, $j=1, \ldots, k$. Let $\phi$ be a state on $\mathscr{A}$ and $P: \mathscr{A} \rightarrow \mathscr{B}$ a completely positive unital map of $\mathscr{A}$ into a finite dimensional abelian $C^{*}$-algebra $\mathscr{B}$ such that there is a state $\mu$ on $\mathscr{B}$ for which $\mu \circ P=\phi$. If $p_{1}, \ldots, p_{r}$ are the minimal projections in $\mathscr{B}$, then there are states $\phi_{i}, i=1, \ldots, r$ of $\mathscr{A}$ such that

$$
P(x)=\sum_{i=1}^{r} \phi_{i}(x) p_{i}, \quad x \in \mathscr{A} .
$$

Since $\mu \circ P=\phi$,

$$
\phi=\sum_{i=1}^{r} \mu\left(p_{i}\right) \phi_{i} .
$$

That is, $\phi$ can be written as a convex combination of the $\phi_{i}$. As in [5], let

$$
\varepsilon_{\mu}(P)=\sum \mu\left(p_{i}\right) S\left(\phi \mid \phi_{i}\right) .
$$


The entropy defect is given by

$$
S_{\mu}(P)=S(\mu)-\varepsilon_{\mu}(P)
$$

where $S(\mu)=-\sum_{i=1}^{r} \mu\left(p_{i}\right) \log \mu\left(p_{i}\right)$ is the entropy of $\mu$.

Let $\mathscr{B}_{j}, j=1, \ldots, k$ be a $C^{*}$-subalgebras of $\mathscr{B}$ and let $E_{j}: \mathscr{B} \rightarrow \mathscr{B}_{j}$ a $\mu$-invariant conditional expectation. Then the quadruple $\left(\mathscr{B}, E_{j}, P, \mu\right)$ is called an abelian model for $\left(\mathscr{A}, \phi, \gamma_{1}, \ldots, \gamma_{k}\right)$ and its entropy is defined to be

$$
S\left(\left.\mu\right|_{\substack{j \\ \mathscr{B}_{j}}}\right)-\sum_{j} S_{\mu}\left(\varrho_{j}\right)
$$

where $\varrho_{j}=E_{j} \circ P \circ \gamma_{j}: \mathscr{N}_{j} \rightarrow \mathscr{B}_{j}$ is a completely positive map from $\mathscr{N}_{j}$ to $\mathscr{B}_{j}$. The supremum of the entropies of all such abelian models is denoted by

$$
H_{\phi}\left(\gamma_{1}, \ldots, \gamma_{k}\right) \text {. }
$$

If $\theta$ is $\phi$-invariant automorphism of $\mathscr{A}$, let $\gamma: \mathscr{N} \rightarrow \mathscr{A}$ be a completely positive unital map of a finite dimensional $C^{*}$-algebra $\mathscr{N}$ to $\mathscr{A}$, and denote by

$$
h_{\phi, \theta}(\gamma)=\lim _{k \rightarrow \infty} \frac{1}{k} H_{\phi}\left(\gamma, \theta \circ \gamma, \ldots, \theta^{k-1} \circ \gamma\right) .
$$

The dynamical entropy of $\theta$ with respect to $\phi$ is defined by

$$
h_{\phi}(\theta)=\sup _{\gamma} h_{\phi, \theta}(\gamma) \text {. }
$$

For the details, we refer the reader to [5].

We collect some useful properties of $H_{\phi}$ from [5]:

Proposition 2.1 [5, Proposition III.6]. a) Let $\theta_{j}: \mathscr{N}_{j}^{\prime} \rightarrow \mathcal{N}_{j}$ be completely positive unital maps, then

$$
H_{\phi}\left(\gamma_{1} \circ \theta_{1}, \ldots, \gamma_{k} \circ \theta_{k}\right) \leqq H_{\phi}\left(\gamma_{1}, \ldots, \gamma_{k}\right) .
$$

Equality holds if $\mathscr{N}_{j} \subset \mathscr{N}_{j}^{\prime}$ and $\theta_{j}$ is a conditional expectation for all $j$.

b) $H_{\phi}\left(\gamma_{1}, \ldots, \gamma_{k}\right)$ depends only upon the set $\left\{\gamma_{1}, \ldots, \gamma_{k}\right\}=X$, that is $H(\gamma, \gamma)=H(\gamma)$.

c) With the notation of b) one has

$$
\max \left\{H_{\phi}(X), H_{\phi}(Y)\right\} \leqq H_{\phi}(X \cup Y) \leqq H_{\phi}(X)+H_{\phi}(Y) .
$$

Proposition 2.2 [5, Proposition IV.3]. Let $\mathscr{A}$ be a unital $C^{*}$-algebra, $\phi$ a state and $\mathscr{N}_{j}, j=1, \ldots, k$ be finite dimensional $C^{*}$-algebras, $\gamma_{j}, \gamma_{j^{\prime}}$ be completely positive unital maps from $\mathscr{N}_{j}$ to $\mathscr{A}$. Let $d$ be the max of the dimensions of the $\mathscr{N}_{j}^{\prime}$ s and $\varepsilon=\max _{j}\left\|\gamma_{j}-\gamma_{j}^{\prime}\right\|$. Then the inequality

$$
\left|H_{\phi}\left(\left(\gamma_{j}\right)_{j=1, \ldots, k}\right)-H_{\phi}\left(\left(\gamma_{j}^{\prime}\right)_{j=1, \ldots, k}\right)\right| \leqq 6 k \varepsilon\left(\frac{1}{2}+\ln \left(1+d \varepsilon^{-1}\right)\right)
$$

holds.

In order to be able to compute $h_{\phi}(\theta)$ it is necessary to have an analogue of the Kolmogorov-Sinai theorem for the ordinary entropy of automorphisms. The following is the KS-type theorem obtained in [5]: 
Theorem 2.3 [5, Corollary V.4]. Let $\mathscr{A}=\bar{\bigcup}_{n} \mathscr{A}_{n}$ be an AF-algebra, then for any state $\phi$ on $\mathscr{A}$ and automorphism $\theta$ with $\phi \circ \theta=\phi$, one has

$$
h_{\phi}(\theta)=\lim _{n \rightarrow \infty} h_{\phi, \theta}\left(\tau_{n}\right) \text {, }
$$

where $\tau_{n}: \mathscr{A}_{n} \rightarrow \mathscr{A}$ is the homomorphism of inclusion.

The following result show that the entropy of an automorphism $\theta$ of a nuclear $C^{*}$-algebra $\mathscr{A}$ with invariant state $\phi$ determines the entropy of the corresponding automorphism of the von-Neumann algebra $\pi_{\phi}(\mathscr{A})^{\prime \prime}$, the weak closure of $\mathscr{A}$ in the GNS construction of $\phi$ :

Theorem 2.4 [5, Theorem VII.2]. Let $\mathscr{A}$ be a nuclear $C^{*}$-algebra, $\phi$ a state on $\mathscr{A}, \theta$ an automorphism with $\phi \circ \theta=\phi$. Let $\mathscr{M}=\pi_{\phi}(\mathscr{A})^{\prime \prime}, \bar{\phi}, \bar{\theta}$ the natural extensions of $\phi, \theta$ to $\mathscr{M}$, then

$$
h_{\bar{\phi}}(\bar{\theta})=h_{\phi}(\theta) \text {. }
$$

For the proofs we refer to [5].

\section{Dynamical Entropy of Quasi-local Algebras: Main Results}

In this section we state our main results and then give some examples for which our results can be applied. We work within the framework of quantum statistical mechanical systems which we start from strictly local algebras $\mathscr{A}_{A}$ indexed by bounded regions $\Lambda$ in $\mathbf{R}^{v}$ (or $\mathbf{Z}^{v}$ ). The norm closure of $\bigcup_{\Lambda} \mathscr{A}_{\Lambda}$ is the $C^{*}$-algebra $\mathscr{A}$ of quasi-local operators. For the general definition of quasi-local algebras indexed by directed sets, we refer reader to [1]. Let $\mathscr{A}$ be a quasi-local algebra whose generating net $\left\{\mathscr{A}_{A}\right\}$ is formed of von-Neumann algebras $\mathscr{A}_{A}$ in separable Hilbert spaces $\mathscr{H}_{A}$. A state $\phi$ on $\mathscr{A}$ is said to be locally normal if $\phi$ is normal in restriction to each $\mathscr{A}_{\Lambda}$. Then $\phi$ in restriction to each $\mathscr{A}_{\Lambda}$ is determined by a density matrix $\varrho_{\Lambda}$ on a Hilbert space $\mathscr{H}_{\Lambda}$.

We list some assumption on quasi-local algebras and then state the main result (Theorem 3.2).

Assumption 3.1. Let $\left(\mathscr{A},\left\{\mathscr{A}_{A}\right\}\right)$ be a quasi-local algebra indexed by bounded open regions $A \subset \mathbf{R}^{v}$ (or $\mathbf{Z}^{v}$ ) and let $\phi$ be a state on $\mathscr{A}$. We assume that the following properties are valid:

(a) For each $\Lambda, \mathscr{A}_{\Lambda}$ is a von Neumann algebra in a separable Hilbert space $\mathscr{H}_{\Lambda}$.

(b) Tensor product property: Let $\Lambda_{1} \subset \Lambda$. Then there exists $\Lambda_{2} \subset A$ such that $\Lambda_{1} \cap \Lambda_{2}=\emptyset$ and $\mathscr{A}_{\Lambda}=\mathscr{A}_{\Lambda_{1}} \otimes \mathscr{A}_{\Lambda_{2}}$.

(c) Locally normal property: $\phi$ is locally normal and for each $\Lambda$ the corresponding density matrix $\varrho_{A}$ belongs to $\mathscr{A}_{\Lambda}$.

Remarks. 1. In the most quantum statistical mechanical systems the equilibrium states obtained via thermodynamic limits of local Gibbs states are turned out to be locally normal $[1,12,13]$.

2. It may be worth commenting that Assumption 3.1 (b) is not satisfied in relativistic quantum field theories. Assumption 3.1 (b) will be used to construct a completely positive unital map $\sigma_{A}$ from $\mathscr{A}$ to $\mathscr{A}_{A}$ which converges to the identity 
map in the pointwise norm topology as $\Lambda$ tends to $\mathbf{R}^{v}$ (or $\mathbf{Z}^{v}$ ). See Lemma 4.1 in Sect. 4 for the details. In order to get such a map or a weaker version of it in quantum field theories, one has to replace Assumption 3.1 (b) with other appropriate property.

3. Our results stated below can easily be generalized to quasi-local algebras indexed by directed sets under the assumptions analogous to those in Assumption 3.1. To avoid the additional notational complications, we restrict ourselves to quasi-local algebras indexed by bounded open regions $\Lambda$ in $\mathbf{R}^{v}$ (or $\mathbf{Z}^{v}$ ).

For a given $\Lambda$, let $\lambda_{i}, i=1,2, \ldots$ be the eigenvalues of the density matrix $\varrho_{\Lambda}$ listed in decreasing order (counting multiplicities), and let $\mathscr{H}_{\Lambda, n}$ be the subspace of $\mathscr{H}_{\Lambda}$ spanned by the eigenvectors corresponding eigenvalues $\lambda_{1}, \lambda_{2}, \ldots, \lambda_{n}$. Denote by $P_{n}$ the projection operator from $\mathscr{H}_{\Lambda}$ to $\mathscr{H}_{\Lambda, n}$ and

$$
\mathscr{A}_{\Lambda, n} \equiv P_{n} \mathscr{A}_{\Lambda} P_{n} \oplus \mathrm{C} \cdot P_{n}^{\perp},
$$

where $P_{n}^{\perp}=\mathbb{1}-P_{n}$. Then from Assumption 3.1 (c) it follows that each $\mathscr{A}_{\Lambda, n}$ is a finite dimensional unital subalgebra of $\mathscr{A}_{\Lambda}$. For each $\Lambda$ and $n$, let $\tau_{\Lambda, n}$ be the embedding map of $\mathscr{A}_{\Lambda, n}$ into $\mathscr{A}_{A}$. Throughout the paper we will adapt the convention that $\Lambda \uparrow \mathbf{R}^{v}$ indicates $\Lambda$ increases to $\mathbf{R}^{v}$ so that $\Lambda$ eventually contains any bounded region of $\mathbf{R}^{v}$.

The following is our main result analogous to Theorem 2.3 for AF-algebra:

Theorem 3.2. Let $\left(\mathscr{A},\left\{\mathscr{A}_{A}\right\}\right)$ be a quasi-local algebra indexed by the bounded regions $\Lambda \subset \mathbf{R}^{v}$ (or $\left.\mathbf{Z}^{v}\right), \theta$ an automorphism on $\mathscr{A}$ and $\phi$ a state on $\mathscr{A}$ invariant under $\theta$. Under the assumptions in Assumption 3.1, one has

$$
h_{\phi}(\theta)=\lim _{\Lambda \uparrow \mathbf{R}^{v}\left(\mathbf{Z}^{v}\right)} \lim _{n \rightarrow \infty} h_{\phi, \theta}\left(\mathscr{A}_{\Lambda, n}\right) .
$$

Here the convention that the subalgebra $\mathscr{A}_{\Lambda, n}$ is standing for the inclusion map $\mathscr{A}_{A, n} \rightarrow \mathscr{A}$ has been used.

Corollary 3.3. Under the assumptions as in Theorem 3.2, one has

$$
h_{\phi}(\theta)=\lim _{\Lambda \uparrow \mathbf{R}^{v}\left(\mathbf{Z}^{v}\right)} \sup _{\substack{\mathcal{N}_{\Lambda} \subset \mathscr{A}_{A}: \\ \mathcal{N}_{\Lambda}: \text { finite }}} h_{\phi, \theta}\left(\mathscr{N}_{\Lambda}\right) .
$$

Proof. The corollary follows from the definition of $h_{\phi}(\theta)$ and Theorem 3.2.

Next let us establish a non-AF version of Theorem 2.4:

Theorem 3.4. Let $(\mathscr{A}, \theta, \phi)$ be a dynamical system and let $\mathscr{M}=\pi_{\phi}(\mathscr{A})^{\prime \prime}, \bar{\phi}, \bar{\theta}$ the natural extensions of $\phi$ and $\theta$ to $\mathscr{M}$. Then under Assumption 3.1 one has

$$
h_{\bar{\phi}}(\bar{\theta})=h_{\phi}(\theta) \text {. }
$$

Proof. In the proof of Theorem 2.4 in [5], the nuclearity of $\mathscr{A}$ is not used and so the same method as that used in the proof of Theorem 2.4 in [5] can be applied to our case. For the details, we refer to [5].

It may be worth to give some examples of quasi-local algebras satisfying Assumption 3.1 (a), (b).

Example 3.5. (Unbounded) Quantum Lattice Systems: For each site $x \in \mathbf{Z}^{v}$, one assigns a separable Hilbert space $\mathscr{H}_{x} \cong \mathscr{H}$. For each finite $\Lambda \subset \mathbf{Z}^{v}$, let $\mathscr{H}_{\Lambda}$ be the 
tensor product space $\mathscr{H}_{\Lambda}=\otimes_{x \in \Lambda} \mathscr{H}_{\{x\}}$. The local $C^{*}$-algebra $\mathscr{A}_{\Lambda}$ is then defined by $\mathscr{A}_{\Lambda}=\mathscr{L}\left(\mathscr{H}_{\Lambda}\right)$. For $\Lambda_{1} \subset \Lambda$, one has that $\mathscr{A}_{\Lambda}=\mathscr{A}_{\Lambda_{1}} \otimes \mathscr{A}_{\Lambda \backslash \Lambda_{1}}$. Note that if $\mathscr{H}_{x}$ is not finite dimensional, then $\mathscr{A}$ is not an AF-algebra.

Example 3.6. CCR algebra: Let $\tilde{H}$ be a (pre)-Hilbert space equipped with inner product $($,$) and let \sigma(f, g)=\operatorname{Im}(f, g)$ for all $f, g \in \mathscr{H}$. Let $\tilde{\mathscr{A}}$ be a $C^{*}$-algebra generated by non-zero elements $W(f)$, for $f \in \mathscr{\mathscr { H }}$, satisfying

$$
\begin{gathered}
W(-f)=W(f)^{*}, \\
W(f) W(g)=e^{-i \sigma(f, g) / 2} W(f+g) \text { for any } f, g \in \tilde{H} .
\end{gathered}
$$

Then there exists the unique $C^{*}$-algebra $\tilde{\mathscr{A}}=\mathscr{A}(\tilde{\mathscr{H}})$ generated by Weyl operators $W(f)$ up to $*$-isomorphism [1].

For each bounded open set $\Lambda \subset \mathbf{R}^{v}$, let $\mathscr{A}_{\Lambda}$ be the CCR-algebra generated by $W(f), f \in L^{2}(\Lambda)$. Then $\left(\mathscr{A},\left\{\mathscr{A}_{\Lambda}\right\}\right)$ is a quasi-local algebra indexed by bounded open set $\Lambda \subset \mathbf{R}^{v}$. Note that for each $\Lambda \subset \mathbf{R}^{v}, \mathscr{A}_{\Lambda}$ is *-isomorphic to $\mathscr{L}\left(\mathscr{F}_{\Lambda}\right)$, where $\mathscr{F}_{\Lambda}$ is the symmetric Fock space [1]. Since $L^{2}(\Lambda)=L^{2}\left(\Lambda_{1}\right) \oplus L^{2}\left(\Lambda_{2}\right)$ for $\Lambda_{1} \subset \Lambda$, where $\Lambda_{2}=\operatorname{int}\left(\Lambda \backslash \Lambda_{1}\right)$, it follows that $\mathscr{A}_{\Lambda}=\mathscr{A}_{\Lambda_{1}} \otimes \mathscr{A}_{\Lambda_{2}}$.

Finally we remark that CAR-algebra over $L^{2}\left(\mathbf{R}^{v}\right)$ is isomorphic to $\otimes M_{2}(\mathbf{C})_{n}$, and so Assumption 3.1 (b) is satisfied if one chooses the index set $I=\{I: I \subset \mathbf{N}, I:$ finite $\}$. Note that CAR-algebra is AF-algebra and so the CNTtheory [5] can be applied directly.

\section{Proof of the Main Result}

In this section we prove Theorem 3.2. The proof is rather lengthy and so we divide the proof into several parts. We first establish some technical lemmas.

Lemma 4.1. Let $\left(\mathscr{A},\left\{\mathscr{A}_{A}\right\}\right)$ be a quasi-local algebra satisfying Assumption 3.1 (b), and let $\tau_{A}: \mathscr{A}_{\Lambda} \rightarrow \mathscr{A}$ be the embedding maps. Then for each bounded region $\Lambda \subset \mathbf{R}^{v}$ (or $\mathbf{Z}^{v}$ ) there is a completely positive map $\sigma_{\Lambda}: \mathscr{A} \rightarrow \mathscr{A}_{\Lambda}$ such that

$$
\lim _{\Lambda \uparrow \mathbf{R}^{v}\left(\mathbf{Z}^{v}\right)}\left\|\tau_{\Lambda} \circ \sigma_{\Lambda}(x)-x\right\|=0 .
$$

for any $x \in \mathscr{A}$.

Proof. Recall that the quasi-local algebra $\mathscr{A}$ is defined to be the norm closure of $\bigcup_{\Lambda^{\prime}} \mathscr{A}_{\Lambda^{\prime}}$. First we will define $\sigma_{\Lambda}$ on the dense subset $\bigcup_{\Lambda^{\prime}} \mathscr{A}_{\Lambda^{\prime}}$ as follows. For $x \in \mathscr{A}_{\Lambda}, \sigma_{\Lambda}(x)=x$. If $x$ is not in $\mathscr{A}_{\Lambda}$, there exists $\Lambda^{\prime}$ such that $x \in \mathscr{A}_{\Lambda^{\prime}}$ and $\Lambda \subset \Lambda^{\prime}$. By assumption $3.1(\mathrm{~b})$, there exists $\Lambda^{\prime \prime} \subset \Lambda^{\prime}$ such that at $x \in \mathscr{A}_{\Lambda^{\prime}}$ and $\Lambda \subset \Lambda^{\prime}$. By Assumption 3.1(b), there exists $\Lambda^{\prime \prime} \subset \Lambda^{\prime}$ such that $\Lambda \cap \Lambda^{\prime \prime}=\emptyset$ and $\mathscr{A}_{\Lambda^{\prime}}=\mathscr{A}_{\Lambda} \otimes \mathscr{A}_{\Lambda^{\prime \prime}}$. Since $\left\{y \otimes z \mid y \in \mathscr{A}_{\Lambda}, z \in \mathscr{A}_{\Lambda^{\prime \prime}}\right\}$ generates $\mathscr{A}_{\Lambda^{\prime}}$, it suffices to define $\sigma_{\Lambda}(x)$ only of the form $x=y \otimes z$, where $y \in \mathscr{A}_{\Lambda}$ and $z \in \mathscr{A}_{\Lambda^{\prime \prime}}$. Let $\phi$ be a state on $\mathscr{A}$. We define $\sigma_{\Lambda}(y \otimes z)=\phi(z) y$. By the linearity $\sigma_{\Lambda}$ extends to $\bigcup_{\Lambda^{\prime}} \mathscr{A}_{\Lambda^{\prime}}$.

By using a corollary of the Hahn-Banach theorem it is easy to show that $\sigma_{\Lambda}$ is a contraction. Hence $\sigma_{A}$ extends to $\mathscr{A}$ by continuity. By the above construction $\sigma_{\Lambda}: \mathscr{A} \rightarrow \mathscr{A}_{\Lambda}$ is a unital conditional expectation. 
We now prove the convergence. For any $x \in \mathscr{A}$ and $\varepsilon>0$, there exists $\Lambda^{\prime}$ such that there exists $x^{\prime} \in \mathscr{A}_{\Lambda^{\prime}}$ with $\left\|x-x^{\prime}\right\|<\varepsilon / 2$. Notice that $\tau_{\Lambda^{\circ}} \sigma_{\Lambda}\left(x^{\prime}\right)=x^{\prime}$ for $\Lambda^{\prime} \subset \Lambda$. For given $x \in \mathscr{A}$ and $\varepsilon>0$ we choose $\Lambda^{\prime}$ and $x^{\prime} \in \mathscr{A}_{\Lambda^{\prime}}$ as above. Then for any $\Lambda$ with $\Lambda^{\prime} \subset \Lambda$ it follows that

$$
\left\|\tau_{\Lambda} \circ \sigma_{\Lambda}(x)-x\right\| \leqq\left\|\tau_{\Lambda} \circ \sigma_{\Lambda}\left(x-x^{\prime}\right)\right\|+\left\|x-x^{\prime}\right\| \leqq 2\left\|x-x^{\prime}\right\|<\varepsilon .
$$

This proves the lemma completely.

The following is a consequence of Proposition 2.2 and Lemma 4.1:

Lemma 4.2. Let $\left(\mathscr{A},\left\{\mathscr{A}_{\Lambda}\right\}\right)$ be a quasi-local algebra as in Lemma 4.1, $\theta$ an automorphism on $\mathscr{A}$ and $\phi$ a state on $\mathscr{A}$ invariant under $\theta$. Then one has

$$
h_{\phi}(\theta)=\lim _{\Lambda \uparrow \mathbf{R}^{v}\left(\mathbf{Z}^{v}\right)} \sup _{\substack{\gamma_{\Lambda}: \mathcal{N} \rightarrow \mathscr{A}_{\Lambda}, \mathscr{N}: \text { finite }}} h_{\phi, \theta}\left(\tau_{\Lambda} \circ \gamma_{\Lambda}\right),
$$

where $\tau_{\boldsymbol{A}}: \mathscr{A}_{\Lambda} \rightarrow \mathscr{A}$ is the inclusion map.

Proof. Let $\tau_{\Lambda}$ and $\sigma_{\Lambda}$ be the completely positive unital maps defined in Lemma 4.1, and let $\gamma^{\prime}: \mathscr{N} \rightarrow \mathscr{A}$ be a completely positive unital map from a finite dimensional algebra $\mathscr{N}$ to $\mathscr{A}$. Put $\gamma_{\Lambda}^{\prime}=\tau_{\Lambda} \circ \sigma_{\Lambda} \circ \gamma^{\prime}$. Then

$$
\lim _{\Lambda \uparrow \mathbf{R}^{v}\left(\mathbf{Z}^{v}\right)}\left\|\gamma_{\Lambda}^{\prime}-\gamma^{\prime}\right\|=0
$$

since by Lemma $4.1 \gamma_{A}^{\prime}$ 's are contractions which converge pointwise in norm to $\gamma^{\prime}$ on the finite dimensional algebra $\mathscr{N}$. Thus Proposition 2.2 shows that

$$
\lim _{\Lambda \uparrow \mathbf{R}^{v}\left(\mathbf{Z}^{v}\right)} h_{\phi, \theta}\left(\gamma_{\Lambda}^{\prime}\right)=h_{\phi, \theta}\left(\gamma^{\prime}\right) .
$$

Thus we use Proposition 2.1 (a) to obtain that

$$
\begin{aligned}
h_{\phi}(\theta) & =\sup _{\gamma^{\prime}: \mathscr{N} \rightarrow \mathscr{A}} h_{\phi, \theta}\left(\gamma^{\prime}\right) \\
& =\sup _{\gamma^{\prime}} \lim _{\Lambda \uparrow \mathbf{R}^{v}\left(\mathbf{Z}^{v}\right)} h_{\phi, \theta}\left(\gamma_{\Lambda}^{\prime}\right) \\
& \leqq \lim _{\Lambda \uparrow \mathbf{R}^{v}\left(\mathbf{Z}^{v}\right)} \sup _{\gamma^{\prime}} h_{\phi, \theta}\left(\gamma_{\Lambda}^{\prime}\right) \\
& \left.\leqq \lim _{\Lambda \uparrow \mathbf{R}^{v}\left(\mathbf{Z}^{v}\right)} \sup _{\gamma_{\Lambda}: \mathscr{N} \rightarrow \mathscr{A}_{\Lambda}} h_{\phi, \theta} \tau_{\Lambda} \circ \gamma_{\Lambda}\right) \\
& \leqq h_{\phi}(\theta) .
\end{aligned}
$$

This proves the lemma.

Let $\phi$ be a state on a $C^{*}$-algebra $\mathscr{A}$. For any positive element $x$ in the commutant $\pi_{\phi}(\mathscr{A})^{\prime}$, we write

$$
\phi_{x}(a)=\phi(x a), \quad \hat{\phi}_{x}(a)=\phi(x a) / \phi(x) .
$$

Then $\hat{\phi}_{x}$ is a state on $\mathscr{A}$.

Proposition 4.3. Let $\left(\mathscr{A},\left\{\mathscr{A}_{A}\right\}\right)$ be a quasi-local algebra, $\theta$ an automorphism on $\mathscr{A}$ and $\phi$ a state on $\mathscr{A}$ invariant under $\theta$. Assume that the conditions in Assumption 3.1 are satisfied. Let $\mathscr{A}_{A, n}$ be the finite dimensional subalgebra of $\mathscr{A}_{A}$ defined in (3.1). Then for a given completely positive unital map $\gamma_{A}: \mathscr{N} \rightarrow \mathscr{A}_{A}$ from a finite dimensional algebra $\mathscr{N}$ to $\mathscr{A}_{A}$, there exist completely positive unital maps $\sigma_{\Lambda, n}: \mathscr{A}_{\Lambda} \rightarrow \mathscr{A}_{\Lambda, n}$ such that the following property holds: For any $\varepsilon>0$ there exists 
$n_{0} \in \mathbf{N}$ such that for $n \geqq n_{0}, k \in \mathbf{Z}$ and for any finite sequence $\left\{x_{1}, \ldots, x_{l}\right\}$ of positive elements in $\pi_{\phi}(\mathscr{A})^{\prime}$ with $\sum x_{i}=1$ the bound

$$
\sum_{i=1}^{l}\left\|\phi_{x_{i}} \circ \theta^{k} \circ \tau_{\Lambda} \circ \tau_{\Lambda, n} \circ \sigma_{\Lambda, n} \circ \gamma_{\Lambda}-\phi_{x_{i}} \circ \theta^{k} \circ \tau_{\Lambda} \circ \gamma_{\Lambda}\right\|^{2} \phi\left(x_{i}\right)^{-1}<\varepsilon
$$

holds, where $\tau_{\Lambda, n}: \mathscr{A}_{\Lambda, n} \rightarrow \mathscr{A}_{\Lambda}$ and $\tau_{\Lambda}: \mathscr{A}_{\Lambda} \rightarrow \mathscr{A}$ are the embedding maps.

Proof. We first construct $\sigma_{\Lambda, n}: \mathscr{A}_{\Lambda} \rightarrow \mathscr{A}_{\Lambda, n}$. Let $P_{n}$ be the projection from $\mathscr{H}_{\Lambda}$ to $\mathscr{H}_{\Lambda, n}$ defined in the above of (3.1). Then $P_{n} \rightarrow 1$ strongly as $n \rightarrow \infty$. Define $\sigma_{\Lambda, n}: \mathscr{A}_{\Lambda} \rightarrow \mathscr{A}_{\Lambda, n}$ by

$$
\sigma_{\Lambda, n}(y)=P_{n} y P_{n}+\left(\phi\left(P_{n}^{\perp} y P_{n}^{\perp}\right) / \phi\left(P_{n}^{\perp}\right)\right) P_{n}^{\perp}
$$

for any $y \in \mathscr{A}_{\Lambda}$. Then $\sigma_{n}(1)=P_{n}+P_{n}^{\perp}=1$. A direct computation shows that $\sigma_{\Lambda, n}\left(x_{1} a x_{2}\right)=x_{1} \sigma_{\Lambda, n}(a) x_{2}$ for $x_{1}, x_{2} \in \mathscr{A}_{\Lambda, n}, a \in \mathscr{A}_{\Lambda}$. Therefore $\sigma_{\Lambda, n}$ is a unital conditional expectation.

Next we prove the bound in the proposition. For a convenience we write that for any $x \in \pi_{\phi}(\mathscr{A})^{\prime}, 0 \leqq x \leqq 1$,

$$
\begin{gathered}
\phi_{x}(\Lambda, j)=\phi \circ x \circ \theta^{j} \circ \tau_{\Lambda} \circ \gamma_{\Lambda}, \\
\phi_{x}(\Lambda, j, n)=\phi \circ x \circ \theta^{j} \circ \tau_{\Lambda} \circ \tau_{\Lambda, n} \circ \sigma_{\Lambda, n} \circ \gamma_{\Lambda} .
\end{gathered}
$$

From (4.3) and the invariance of $\phi$ under $\theta$ it follows that

$$
\begin{aligned}
\phi_{x_{i}}(\Lambda, j, n)(a)= & \phi\left(\theta^{-j}\left(x_{i}\right) P_{n} \gamma_{\Lambda}(a) P_{n}\right) \\
& +\phi\left(\theta^{-j}\left(x_{i}\right) P_{n}^{\perp}\right) \phi\left(P_{n}^{\perp} \gamma_{\Lambda}(a) P_{n}^{\perp}\right) \phi\left(P_{n}^{\perp}\right)^{-1} \\
\equiv & I_{1}+I_{2}, \\
\phi_{x_{i}}(\Lambda, j)(a)= & \phi\left(\theta^{-j}\left(x_{i}\right)\left(P_{n}+P_{n}^{\perp}\right) \gamma_{\Lambda}(a)\left(P_{n}+P_{n}^{\perp}\right)\right) \\
= & \phi\left(\theta^{-j}\left(x_{i}\right) P_{n} \gamma_{\Lambda}(a) P_{n}\right)+\phi\left(\theta^{-j}\left(x_{i}\right) P_{n} \gamma_{\Lambda}(a) P_{n}^{\perp}\right) \\
& +\phi\left(\theta^{-j}\left(x_{i}\right) P_{n}^{\perp} \gamma_{\Lambda}(a) P_{n}\right)+\phi\left(\theta^{-j}\left(x_{i}\right) P_{n}^{\perp} \gamma_{\Lambda}(a) P_{n}^{\perp}\right) \\
\equiv & I_{1}+I I_{1}+I I_{2}+I I_{3} .
\end{aligned}
$$

We use the Schwarz inequality and the facts that $P_{n}, P_{n}^{\perp} \in \mathscr{A}_{\Lambda}$ and $\theta^{-j}\left(x_{i}\right) \in \pi_{\phi}(\mathscr{A})^{\prime}$ to obtain that

$$
\begin{aligned}
& \left|I_{2}\right| \leqq \phi\left(\theta^{-j}\left(x_{i}\right) P_{n}^{\perp}\right)\left\|\gamma_{\Lambda}(a)\right\| \\
& \leqq \phi\left(x_{i}\right)^{1 / 2} \phi\left(\theta^{-j}\left(x_{i}\right) P_{n}^{\perp}\right)^{1 / 2}\left\|\gamma_{A}\right\|\|a\|, \\
& \left|I I_{1}\right|+\left|I I_{2}\right| \leqq 2 \phi\left(\theta^{-j}\left(x_{i}\right) P_{n}\right)^{1 / 2} \phi\left(P_{n}^{\perp} \theta^{-j}\left(x_{i}\right) \gamma_{\Lambda}(a)^{*} \gamma_{\Lambda}(a) P_{n}^{\perp}\right)^{1 / 2} \\
& \leqq 2 \phi\left(x_{i}\right)^{1 / 2} \phi\left(\theta^{-j}\left(x_{i}\right) P_{n}^{\perp}\right)^{1 / 2}\left\|\gamma_{A}\right\|\|a\|, \\
& \left|I I_{3}\right| \leqq \phi\left(\theta^{-j}\left(x_{i}\right) P_{n}^{\perp}\right)\left\|\gamma_{\Lambda}\right\|\|a\| \\
& \left.\leqq \phi\left(x_{i}\right)^{1 / 2} \phi\left(\theta^{-j}\left(x_{i}\right) P_{n}^{\perp}\right)^{1 / 2}\right)\left\|\gamma_{A}\right\|\|a\| \text {. }
\end{aligned}
$$


Thus the above bounds imply that since $\sum_{i} x_{i}=1$,

$$
\begin{aligned}
\sum_{i} & \left\|\phi_{x_{i}}(\Lambda, j, n)-\phi_{x_{i}}(\Lambda, j)\right\|^{2} \phi\left(x_{i}\right)^{-1} \\
& \leqq \sum_{i} 16 \phi\left(\theta^{-j}\left(x_{i}\right) P_{n}^{\perp}\right)\left\|\gamma_{\Lambda}\right\|^{2} \\
& =16\left\|\gamma_{\Lambda}\right\|^{2} \phi\left(P_{n}^{\perp}\right) \\
& =16\left\|\gamma_{\Lambda}\right\|^{2} \sum_{m=n+1}^{\infty} \lambda_{m} .
\end{aligned}
$$

The above tends to zeros as $n \rightarrow \infty$. This proved the bound in the proposition.

Proposition 4.4. Under the assumptions as in Proposition 4.3, one has that for a given completely positive map $\gamma_{\Lambda}: \mathscr{N} \rightarrow \mathscr{A}_{\Lambda}$ and $\varepsilon>0$, there exists $n_{0} \in \mathbf{N}$ such that for $n \geqq n_{0}$,

$$
\begin{aligned}
& \mid H_{\phi}\left(\left(\theta^{i} \circ \tau_{\Lambda} \circ \gamma_{\Lambda}\right)_{i=0,1, \ldots, k-1}\right) \\
& \quad-H_{\phi}\left(\left(\theta^{i} \circ \tau_{\Lambda} \circ \tau_{\Lambda, n} \circ \sigma_{\Lambda, n} \circ \gamma_{\Lambda}\right)_{i=0,1, \ldots, k-1}\right) \mid \leqq k \varepsilon .
\end{aligned}
$$

Proof. We will use the method similar to that used in the proof of Lemma 2.1 of [11]. We remark that an abelian model $\left(\mathscr{B}, \mu, \mathscr{B}_{j}\right)_{j=1, \ldots, k}$ for $\left(\mathscr{A}, \phi, \theta^{j} \circ \gamma\right)_{j=1, \ldots, k}$ is equivalent to a decomposition of the unit $\left\{x_{I}\right\}_{I=i_{1} \ldots i_{k}}$ such that $x_{I} \in \pi_{\phi}(\mathscr{A})^{\prime}, x_{I} \geqq 0$, $\sum_{I} x_{I}=1$. Denote that

$$
x_{i_{m}}=\sum_{\substack{i_{1} \ldots i_{k} \\ i_{m}: \text { fixed }}} x_{I}, \quad \phi_{i_{m}}(a)=\phi\left(x_{i_{m}} a\right), \quad \hat{\phi}=\phi / \phi(1) .
$$

Then $H_{\phi}$ can be written as in [5]

$$
\begin{aligned}
& H_{\phi}\left(\left(\theta^{i} \circ \tau_{\Lambda} \circ \gamma_{\Lambda}\right)_{i=0,1, \ldots, k-1}\right) \\
& =\sup _{\Sigma \phi_{(i)}=\phi}\left[\sum_{(i)} \eta\left(\phi_{(i)}(1)\right)-\sum_{m=0}^{k-1} \sum_{i_{m}} \eta\left(\phi_{i_{m}}(1)\right)\right. \\
& \left.\quad+\sum_{m=0}^{k-1}\left\{S\left(\phi \circ \theta^{m} \circ \tau_{\Lambda} \circ \gamma_{\Lambda}\right)-\sum_{i_{m}} \phi_{i_{m}}^{(m)}(1) S\left(\hat{\phi}_{i_{m}}^{(m)} \circ \theta^{m} \circ \tau_{\Lambda} \circ \gamma_{\Lambda}\right)\right\}\right],
\end{aligned}
$$

where $\eta(x)=-x \log x$.

In order to show the method of the proof we first show that for $n \geqq n_{0}$,

$$
\left|H_{\phi}\left(\tau_{\Lambda} \circ \gamma_{\Lambda}\right)-H_{\phi}\left(\tau_{\Lambda} \circ \tau_{\Lambda, n} \circ \sigma_{\Lambda, n} \circ \gamma_{\Lambda}\right)\right|<\varepsilon
$$

For a notational simplification, we use the following abbreviated notations:

$$
\begin{array}{cc}
\phi(\Lambda)=\phi \circ \tau_{\Lambda} \circ \gamma_{\Lambda}, & \phi_{j}(\Lambda)=\phi_{j} \circ \tau_{\Lambda} \circ \gamma_{\Lambda}, \\
\phi(\Lambda, n)=\phi \circ \tau_{\Lambda} \circ \tau_{\Lambda, n} \circ \sigma_{\Lambda, n} \circ \gamma_{\Lambda}, & \phi_{j}(\Lambda, n)=\phi_{j} \circ \tau_{\Lambda} \circ \tau_{\Lambda, n} \circ \sigma_{\Lambda, n} \circ \gamma_{\Lambda}, \\
\hat{\phi}_{j}(\Lambda)=\phi_{j}(\Lambda) / \phi_{j}(1), & \hat{\phi}_{j}(\Lambda, n)=\phi_{j}(\Lambda, n) / \phi_{j}(1) .
\end{array}
$$

We assume that $\left\{x_{i}\right\}$ define for $H_{\phi}\left(\tau_{\Lambda} \circ \gamma_{\Lambda}\right)$ a (close to) optimal decomposition, i.e.

$$
H_{\phi}\left(\tau_{\Lambda} \circ \gamma_{\Lambda}\right) \leqq S(\phi(\Lambda))-\sum_{i} \phi_{i}(1) S\left(\hat{\phi}_{i}(\Lambda)\right)+\varepsilon
$$


For this decomposition one has

$$
H_{\phi}\left(\tau_{\Lambda} \circ \tau_{\Lambda, n} \circ \sigma_{\Lambda, n} \circ \gamma_{\Lambda}\right) \geqq S(\phi(\Lambda, n))-\sum_{i} \phi_{i}(1) S\left(\hat{\phi}_{i}(\Lambda, n)\right) .
$$

By Proposition 4.3 we have that for $n \geqq n_{0}$,

$$
\sum_{i}\left\|\phi_{i}(\Lambda)-\phi_{i}(\Lambda, n)\right\|^{2} \phi_{i}(1)^{-1}<\varepsilon .
$$

As in [11], we write

$$
\left\|\phi_{i}(\Lambda)-\phi_{i}(\Lambda, \mathrm{n})\right\|^{2} \phi_{i}(1)^{-1}=\varepsilon_{i} \phi_{i}(1)
$$

and decompose the sum into two parts:

$$
I=\left\{i: \varepsilon_{i}<\varepsilon^{2 / 3}\right\}, \quad I_{c}=\left\{i: \varepsilon_{i} \geqq \varepsilon^{2 / 3}\right\} .
$$

Then it follows that

$$
\sum_{I_{c}} \phi_{i}(1) \leqq \varepsilon^{1 / 3} .
$$

Put $v=\sum_{I_{c}} \phi_{i}$. Then we have that $v(1) \leqq \varepsilon^{1 / 3}$. For $i \in I$, we estimate

$$
\left\|\hat{\phi}_{i}(\Lambda)-\hat{\phi}_{i}(\Lambda, n)\right\|^{2}=\phi_{i}(1)^{-2}\left\|\phi_{i}(\Lambda)-\phi_{i}(\Lambda, n)\right\|^{2} \leqq \varepsilon^{2 / 3} .
$$

By the continuity of entropy in [11] and Proposition 4.3 (with $x_{1}=1$ and $x_{i}=0$ for $i=2,3, \ldots, l)$ it follows that for $i \in I$,

$$
\begin{aligned}
\left|S\left(\hat{\phi}_{i}(\Lambda)\right)-S\left(\hat{\phi}_{i}(\Lambda, n)\right)\right| & \leqq \varepsilon^{1 / 3} \log \left(2 d / \varepsilon^{1 / 3}\right), \\
\mid S(\phi(\Lambda)-S(\phi(\Lambda, n)) \mid & \leqq \varepsilon^{1 / 2} \log \left(2 d / \varepsilon^{1 / 2}\right),
\end{aligned}
$$

where $d$ is the dimension of $\mathscr{N}$. From (4.9), (4.10) and the estimates in the above, we obtain that

$$
\begin{aligned}
& H_{\phi}\left(\tau_{\Lambda} \circ \gamma_{\Lambda}\right)-H_{\phi}\left(\tau_{\Lambda}^{\circ} \circ \tau_{\Lambda, n} \circ \sigma_{\Lambda, n} \circ \gamma_{\Lambda}\right) \\
& \leqq \\
& \quad+\phi(\Lambda))-S(\phi(\Lambda, n)) \\
& \quad+\sum_{i \in I} \phi_{i}(1)\left|S\left(\hat{\phi}_{i}(\Lambda)\right)-S\left(\hat{\phi}_{i}(\Lambda, n)\right)\right| \\
& \quad+\sum_{i \in I_{c}} \phi_{i}(1)\left(\left|S\left(\hat{\phi}_{i}(\Lambda)\right)\right|+\left|S\left(\hat{\phi}_{i}(\Lambda, n)\right)\right|\right)+\varepsilon \\
& \leqq \\
& \varepsilon^{1 / 2} \log \left(2 d / \varepsilon^{1 / 2}\right)+\varepsilon^{1 / 3} \log \left(2 d / \varepsilon^{1 / 3}\right)+2 \varepsilon^{1 / 3} \log (d)+\varepsilon .
\end{aligned}
$$

Here we have used the fact that $|S(\phi)| \leqq\|\phi\| \log (\|\phi\| d)$. By redefining $\varepsilon$ in the above and interchanging the role of $\phi(\Lambda)$ and $\phi(\Lambda, n)$, etc., we proved the bound in (4.7).

The proof of the proposition now follows from Proposition 4.3, (4.6) and the method used in the proof of (4.7). Notice that the sum over $j$ gives $k$ factor in the bound. This proved the proposition completely.

Finally we turn to the proof of our main result:

Proof of Theorem 3.2. From Proposition 4.4 it follows that for a given $\gamma_{\Lambda}: \mathscr{N} \rightarrow \mathscr{A}_{\Lambda}$,

$$
h_{\phi, \theta}\left(\tau_{\Lambda} \circ \gamma_{\Lambda}\right)=\lim _{n \rightarrow \infty} h_{\phi, \theta}\left(\tau_{\Lambda} \circ \tau_{\Lambda, n} \circ \sigma_{\Lambda, n} \circ \gamma_{\Lambda}\right)
$$


We now use the above relation and Proposition 2.1 (a) to conclude that

$$
\begin{aligned}
\lim _{n \rightarrow \infty} h_{\phi, \theta}\left(\tau_{\Lambda} \circ \tau_{A, n}\right) & \leqq \sup _{\gamma_{\Lambda}: \mathcal{S} \rightarrow \mathscr{A}_{\Lambda}} h_{\phi, \theta}\left(\tau_{\Lambda} \circ \gamma_{\Lambda}\right) \\
& \leqq \sup _{\gamma_{\Lambda}: \mathscr{N} \rightarrow \mathscr{A}_{\Lambda}} \lim _{n \rightarrow \infty} h_{\phi, \theta}\left(\tau_{\Lambda} \circ \tau_{\Lambda, n} \circ \sigma_{\Lambda, n} \circ \gamma_{\Lambda}\right) \\
& \leqq \lim _{n \rightarrow \infty} \sup _{\gamma_{\Lambda}: \mathcal{S} \rightarrow \mathscr{A}_{\Lambda}} h_{\phi, \theta}\left(\tau_{\Lambda} \circ \tau_{\Lambda, n} \circ \sigma_{\Lambda, n} \circ \gamma_{\Lambda}\right) \\
& \leqq \lim _{n \rightarrow \infty} h_{\phi, \theta}\left(\tau_{\Lambda} \circ \tau_{\Lambda, n}\right)
\end{aligned}
$$

and so

$$
\lim _{n \rightarrow \infty} h_{\phi, \theta}\left(\tau_{\Lambda} \circ \tau_{\Lambda, n}\right)=\sup _{\gamma_{\Lambda}: \mathscr{N} \rightarrow \mathscr{A}_{\Lambda}} h_{\phi, \theta}\left(\tau_{\Lambda} \circ \gamma_{\Lambda}\right) .
$$

Thus Theorem 3.2 follows from Lemma 4.2 and the above result. This proved the main theorem completely.

\section{A Simple Application}

Recently the dynamical entropies of space translation for the Gibbs state of one dimensional bounded quantum lattice system $[5,11]$ and Bogoliubov automorphisms for the quasifree state of CAR algebra [16] were computed. We expect that these results can be extended to the unbounded quantum lattice system and CCR algebra, and leave it to further study.

As a simple (almost trivial) application of Theorem 3.2 (and Corollary 3.3) let us compute the dynamical entropy of space translations for product states of unbounded quantum lattice systems. As in Example 3.5, we assign for each site $x \in \mathbf{Z}$ a separable Hilbert space $\mathscr{H}_{x} \cong \mathscr{H}$. For each finite $\Lambda \subset \mathbf{Z}$, let

$$
\mathscr{H}_{\Lambda}=\bigotimes_{x \in \Lambda} \mathscr{H}_{x}, \quad \mathscr{A}_{\Lambda}=\bigotimes_{x \in \boldsymbol{x} \Lambda} \mathscr{L}\left(\mathscr{H}_{x}\right)
$$

Let $\varrho_{x} \cong \varrho$ be a density matrix on $\mathscr{H}_{x}$ with eigenvalues $\lambda_{i}, i \in \mathbf{N}$, listed in decreasing order, $\phi_{x}$ the corresponding state on $\mathscr{L}\left(\mathscr{H}_{x}\right)$, and $\phi=\prod_{x \in \mathbf{Z}} \phi_{x}$ the product state on the quasi-local algebra $\mathscr{A}=\left(\bigcup_{\Lambda} \mathscr{A}_{A}\right)^{-}$.

Theorem 5.1. Let $\left(\mathscr{A},\left\{\mathscr{A}_{A}\right\}\right)$ be a quasi-local algebra for a unbounded quantum lattice system indexed by $\Lambda \subset \mathbf{Z}, \theta$ the automorphism on $\mathscr{A}$ given by one step lattice translation, and $\phi$ the product state. Then the equality

holds.

$$
h_{\phi}(\theta)=-\sum_{i} \lambda_{i} \log \lambda_{i}
$$

Proof. Let $\Lambda=\{1,2, \ldots, l\} \subset \mathbf{N}$. For a given finite dimensional subalgebra $\mathscr{N}_{A} \subset \mathscr{A}_{A}$ and $k \in \mathbf{N}$, let $M_{\Lambda, k}$ be the finite dimensional subalgebra of $\mathscr{A}_{\Lambda(k)}, \Lambda(k)=\{1,2, \ldots$, $l+k\}$, generated by $\theta^{j}\left(\mathcal{N}_{A}\right), j=0,1, \ldots, k-1$. Then by the monotonicity of $H_{\phi}$ in Proposition 2.1

$$
\begin{aligned}
H_{\phi}\left(\mathcal{N}_{A}, \theta\left(\mathscr{N}_{A}\right), \ldots, \theta^{k}\left(\mathscr{N}_{A}\right)\right) & \leqq H_{\phi}\left(M_{A, k}, \ldots, M_{A, k}\right) \\
& =H_{\phi}\left(M_{A, k}\right) .
\end{aligned}
$$


We use Proposition 2.1 and Proposition 4.4 to obtain

$$
\begin{aligned}
H_{\phi}\left(M_{\Lambda, k}\right) & \leqq \lim _{n \rightarrow \infty} H_{\phi}\left(\mathscr{A}_{\Lambda(k), n}\right) \\
& \leqq \lim _{n \rightarrow \infty} S_{\phi}\left(\mathscr{A}_{\Lambda(k), n}\right) \\
& =-(l+k) \sum_{i} \lambda_{i} \log \lambda_{i} .
\end{aligned}
$$

Thus by Theorem 3.2 (Corollary 3.3)

$$
h_{\phi}(\theta) \leqq-\sum_{i} \lambda_{i} \log \lambda_{i}
$$

Next we get the lower bound of $h_{\phi}(\theta)$. By the monotonicity of $H_{\phi}$,

$$
\begin{aligned}
& H_{\phi}\left(\mathscr{A}_{\Lambda, n}, \theta\left(\mathscr{A}_{\Lambda, n}\right), \ldots, \theta^{m k-1}\left(\mathscr{A}_{\Lambda, n}\right)\right) \\
& \quad \geqq H_{\phi}\left(\mathscr{A}_{\Lambda, n}, \theta^{m}\left(\mathscr{A}_{\Lambda, n}\right), \ldots, \theta^{m(k-1)}\left(\mathscr{A}_{\Lambda, n}\right)\right) .
\end{aligned}
$$

For $\Lambda=\{1,2, \ldots, l\}$ we choose $m=l$. Since $\phi$ is a product state and $\theta^{m k}\left(\mathscr{A}_{\Lambda, n}\right)$ are pairwise commuting subalgebras, the right-hand side of (5.1) equals to $k H_{\phi}\left(\mathscr{A}_{\Lambda, n}\right)$. Let $\varrho=\sum \lambda_{i} p_{i}$ be the spectral decomposition of the density matrix, $P_{n}=p_{1}+\ldots+p_{n}$ and $P_{n}^{\perp}=\mathbb{1}-P_{n}$. Then $\left\{p_{1}, \ldots, p_{n}, P_{n}^{\perp}\right\}$ defines a decomposition of $\phi_{x}$. A direct computation shows that

$$
H_{\phi}\left(\mathscr{A}_{\Lambda, n}\right) \geqq-l\left(\sum_{i=1}^{n} \lambda_{i} \log \lambda_{i}\right) .
$$

Thus by Theorem 3.2 and the above calculation we obtain

$$
h_{\phi}(\theta) \geqq-\sum_{i} \lambda_{i} \log \lambda_{i}
$$

This proves the theorem completely.

Acknowledgements. The authors are happy to express their gratitude to Prof. H. Narnhofer and Prof. W. Thirring who made valuable comments on the first draft of the paper. This work was supported by the Korean Science Foundation and the Basic Science Research Program, Korean Ministry of Education, 1991.

\section{References}

1. Bratteli, O., Robinson, D.W.: Operator algebras and quantum statistical mechanics, Vols.I and II. Berlin, Heidelberg, New York: Springer (1979)

2. Confeld, I.P., Fomin, S.V., Sinai, Ya.G.: Ergodic theory. Berlin, Heidelberg, New York: Springer 1980

3. Connes, A., Störmer, E.: Acat. Math. 134, 289 (1975)

4. Connes, A.: C.R. Acad. Sci. Paris t. 301 I, 1 (1985)

5. Connes, A., Narnhofer, H., Thirring, W.: Commun. Math. Phys. 112, 691 (1987)

6. Emch, G.: Acta. Phys. Austr. [Suppl.] XV, 79 (1976)

7. Kadison, R.V., Ringrose, J.R.: Fundamentals of the theory of operator algebras, Vols. I, II. New York: Academic Press 1986

8. Kolmogorov, A.N.: Dokl. Akad. Nauk. 119, 861 (1958)

9. Lieb, E.H.: Convex trace functions and the Wigner-Yanase-Dyson conjecture. Adv. Math. 11, 267 (1973) 
10. Narnhofer, H., Thirring, W.: From relative entropy to entropy. Fizika 17, 257 (1985)

11. Narnhofer, H., Thirring, W.: Dynamical entropy and the third law of thermodynamics. Lett. Math. Phys. 15, 261-273 (1988)

12. Park, Y.M.: Quantum statistical mechanics for superstable interactions; Bose-Einstein statistics. J. Stat. Phys. 40, 259 (1984)

13. Park, Y.M.: Quantum statistical mechanics of unbounded continuous spin systems. J. Korean Math. Soc. 22(1), 43-74 (1985)

14. Ruelle, D.: Thermodynamic formalism. Reading, MA: Addison-Wesley 1978

15. Sinai, Ya.G.: Dokl. Akad. Nauk. 124, 768 (1959)

16. Störmer, E., Voiculescu, D.: Entropy of Bogoliubov automorphisms of canonical anticommutation relations. Commun. Math. Phys. 133, 521-542 (1990)

17. Takesaki, M.: Theory of operator algebra. I. Berlin, Heidelberg, New York: Springer 1979

Communicated by A. Connes 
